\title{
Evaluation of Electrical Submersible Pump on Field Production Network Optimization in Niger Delta Oilfield
}

\author{
Anthony Kerunwa', Julian Ubanozie Obibuike1, Ndubuisi Uchechukwu Okereke1, \\ Stephen G. Udeagbara ${ }^{2}$, Angela Nkechinyere Nwachukwu ${ }^{1}$, Stanley Toochukwu Ekwueme ${ }^{1}$ \\ ${ }^{1}$ Department of Petroleum Engineering, Federal University of Technology, Owerri, Nigeria \\ ${ }^{2}$ Department of Chemical and Petroleum Engineering, Afe Babalola University, Ado-Ekiti, Nigeria \\ Email: anthonykerunwa@rocketmail.com, obibuikeubanozie@gmail.com,stanleyekwueme@yahoo.com
}

How to cite this paper: Kerunwa, A., Obibuike, J.U., Okereke, N.U., Udeagbara, S.G., Nwachukwu, A.N. and Ekwueme, S.T. (2022) Evaluation of Electrical Submersible Pump on Field Production Network Optimization in Niger Delta Oilfield. Open Journal of Yangtze Gas and Oil, 7, 26-47.

https://doi.org/10.4236/ojogas.2022.71003

Received: October 4, 2021

Accepted: December 28, 2021

Published: December 31, 2021

Copyright $\odot 2022$ by author(s) and Scientific Research Publishing Inc. This work is licensed under the Creative Commons Attribution International License (CC BY 4.0).

http://creativecommons.org/licenses/by/4.0/

(c) (i) Open Access

\begin{abstract}
Complexity arises when trying to maximize oil productions from fields using Electrical Submersible Pumps (ESP). The complexity increases with the increase in the number of reservoirs and wells in a particular field. Individual well's ESP frequencies have to be constantly updated to ensure optimum oil productions from the field. The choice of the ESP frequency to be used must come from sound engineering decisions which do not come from mere intuition but must be backed up by mathematical models and computer simulations. This study proposes to evaluate field production network optimization on ESP lifted wells using quadratic sequential programming techniques. The optimization approach seeks to determine the ESP frequency for each well that will lead to the maximum field oil production while honouring the field operational constraints. Two reservoirs and five wells were considered. The non-linear optimization problem for the ESP lifted wells in the field was formulated with their boundary conditions. The simulations were performed in Prosper and GAP software. Prosper software was used in building the individual well's ESP models for the five wells in the field. Individual well's model in Prosper was exported to GAP and simulations were run in GAP for the field network system. GAP simulations were run in two cases: case 1 comprises ESP simulation without optimization while case 2 comprises ESP simulation with optimization. For case 1, fixed values of ESP frequency were selected for each well and the GAP software calculates the production rates from the wells in the network accruing from the ESP frequencies inputted. For case 2, there was no input ESP frequency as the GAP software was allowed to calculate based on optimization algorithms, the best suitable ESP frequencies for each well in the field that will lead to the maximum total oil production in the field network
\end{abstract}


while honouring the operational constraint imposed on the systems in the field. From the results, it was realized that at the basis of well, the higher the ESP frequency, the higher the well's production rates. Sensitivities on the effects of separator pressure on production rates show that separator pressures affect the well's productions rates. A reduction in separator pressure from 200 psig to 80 psig led to a $1.69 \%$ increase in field oil rate. Comparison of results for case 1 and case 2 showed that ESP field network simulation with optimization yields had a higher field production rate than ESP field network simulations without optimization. There was an increase in oil rate of $1.16 \%$ and $2.66 \%$ for constraints 1 and 2 when ESP simulation was done with optimization rather than without optimization. Also, simulation with optimization comes with higher pump efficiency than simulation without optimization.

\section{Keywords}

Separator Pressures, Reservoir, Multistage, Centrifugal Pump, Oil Production

\section{Introduction}

The production engineer is often faced with the complex and challenging task of ensuring maximum recovery during the exploitation of mature petroleum assets. Maximum recovery is made possible by utilizing current technologies and practices alongside the consistent and rigorous application of modeling, simulation and optimization [1]. Enhanced knowledge of physical flow phenomena, advanced mathematical techniques and high performance computing are essential factors with the potentials to increase process efficiency and lower operational cost when systematically applied [2] [3]. An oil and gas production system comprises many interconnected components; these include reservoirs, wells, manifolds, pipelines, surface equipment such as pumps, compressors, separators, etc. Given the nature of the production network, an optimization approach is one that guarantees maximum oil recovery from the field at the least operational cost given various operational constraints imposed on the system. When considering production optimization of petroleum assets, two approaches come to mind: the well-based and the field-wide optimization methods [3]. At the well level, individual wells are optimized with the intent to achieve maximum oil recovery from the well. This is achieved by making changes or adjustments to parameters peculiar to a specific well [4] [5] [6]. Conversely, for the field-wide optimization, many complexities exist because of the presence of many wells with often varying parameters in the field. These wells mostly exist in the form of clusters. Thus, there are problems associated with field optimization in a system of well clusters. The individual wells may have varying designs, trajectory, depths, and water cuts [7]. These may affect the common field facilities shared among the wells such as pump pressure requirement, separator capacity, back pressure coming from shared surface lines, separator position in relation to production manifolds, space availability for 
storage of produced fluids especially in offshore locations [8]. Electrical Submersible Pumps (ESPs) have found extensive application in the oil and gas industry as an artificial lift pump [9] [10]. It is mostly utilized for high productivity wells, high water cut oil wells, dewatering of Coal Bed Methane (CBM) wells, heavy oil wells, and deep-sea wells where it is utilized as seafloor boosting method. There is often a great challenge in the management and production of ESP production networks in oilfields. The accurate decision has to be made regarding the update of individual ESP frequencies and shut-in high water-producing wells. Several constraints exist due to interdependence between the wells in the field [11] [12]. There is a great need for production optimization utilizing precise numerically developed models of the production system that will serve as a decision support tool in the determination of the best operating ESP frequency that will achieve the maximum oil production while not neglecting the prevalent operational constraints [13] [14]. In this study, field network optimization of the heavy oilfield is conducted using electrical submersible pumps. The Niger delta field AKUX having two reservoirs with five wells is selected for a case study. The optimization problem is solved using the quadratic sequential programming method by application of non-linear mathematical optimization methodology. Prosper and GAP software in petroleum experts' suites are used for the simulation. Prosper ensures the well-based simulation using ESP while GAP is utilized in the modeling and simulation of the field network optimization.

\section{Literature Review}

[15] [16] classified production system optimization methods into simulationbased, heuristic-based and mathematical programming methods. They agreed that these methods have aided in the design and operation of production systems, rate allocation, reservoir planning and development. Nevertheless, there are areas where these methods fail to yield satisfactory results. These include naturally flowing vertical well and wells with structure specific methodologies. [17] conducted a thorough research relating the differences between production optimization from petroleum engineering and process systems perspectives. Their research enabled them to classify research endeavors into oil field design, oil field operations and integrated field design and operations. They concluded that optimization from petroleum engineering perspective paid more attention to subsurface while optimization from process system perspective paid more attention to surface networks. They recommended that further research should focus on ways to integrate field design and operation; hence helping to capture surface and subsurface complexities. [18] conducted a research where they combined decline curve analysis using real production data with production optimization. They targeted to achieve quick prediction of future operating rates in the field and as well reduce the time requirement in developing detailed numerical reservoir models, and the difficulty of using such models for optimization purposes. Though their approach did not sufficiently capture the fundamental flow physics, they 
argued that it could offer good initial solutions for subsequent large-scale optimization problems with full reservoir simulation. [19] presented a very peculiar solution of field network optimization using ESP. They used Mixed Integer Linear Program approach and formulated a piecewise linearization which enabled them to determine the ESP performance characteristics for the different wells in their production network. They reported fast solution which gave credence to their approach and makes its suitable to be applied in real-time. The work of [20] revealed the challenges surrounding a simulator in an optimization problem. They maintained that most simulators find it difficult to calculate gradients required for quick performance of an optimization process. They proposed a solution to this problem by using simulation based optimization method that considers the complex behaviour of production system components via simulator data approximation. [21] presented a comprehensive discussion on the roles of ESP surveillance to analyze and improve the operation of ESP-lifted wells. They utilized historical and real-time data during startup, operation, shutdown and modeling. [22] developed a system that serves as an advisory aimed to support the production management of Rubiales field based on performing short-term model-based optimization. Their approach involves the application of mathematical optimization techniques on a numerical model of the production network developed in a commercial software. They assumed pseudo-steady state inflow deliverability in formulating their model which was able to solve the flow equilibrium and helped in the computation of the rates, pressure and temperatures along the system. Changing ESP frequencies was used in the optimization process aimed at ensuring maximum oil recovery while honoring multiple operational constraints. They made use of an open source interior-point filter line-search algorithm as the optimization tool. They obtained values for ESP frequencies that maximized oil recovery. However their model was limited by high running time periods.

\subsection{Electrical Submersible Pumps (ESPs)}

ESPs are multistage centrifugal pumps used as artificial lift pumps to lift fluids from the wellbore to the surface. They are placed several metres below inside the well tubing. The operation of ESP is achieved by making adjustments to the rotational speed of the pump and the production choke. These constitute the two variables that can be manipulated in the ESP operation in a well [23]. In ESP operation, the size of the pump defines the maximum capacity of the ESP at a given rotational speed. Also, the size of the production casing determines the maximum pump size and rate of the ESP to be fitted. Also, the rotational speed of the ESP depends on the alternating speed (frequency) of the current supply [24]. Variable speed drives are utilized for adjusting and controlling frequency thereby enabling the control of production rates over a range for each pump. ESPs achieve highest performance when the fluid lifted is entirely liquid. Because ESPs are centrifugal pumps, the presence of gas in the lifted fluid impacts on its per- 
formance. The presence of gas deteriorates ESP lifting capacity and pump blockage may occur at higher volumetric gas fractions [25]. However, ESP has the capacity to handle free gas in fluid up to $20 \%$ volume fraction without separation of gas or without the presence of gas handling equipment [24] [25]. Higher volume fraction of gas in fluid lifted by ESP can be detrimental to pump performance, life and gas handling equipment may be required to separate the gas [25].

\subsection{ESP Optimization in a Field of Clustered Wells}

A field may have several ESP lifted wells producing to a common manifold. In such a case, the operation of one ESP in a well affects the operation of other ESPs lifted wells in the field due to coupling through the pressure of the manifold. This is because the wells share a common manifold. Furthermore, other differences such as fluid viscosity, reservoir inflow conditions and the available power may also change, which affects the optimal operation of the pump. The volume of fluid production from each well in the field is varied by changing the speed of the ESP pump. The ESP speed can be changed by changing the electrical frequency of the driving motor. Aside the ESP, the production from the reservoir can be regulated by the chokes. Usually ESP operates at its normal frequency usually 60 Hz. Most times, operating at this frequency does not yield optimal results. For instance if the separator capacity is low, running ESP at $60 \mathrm{~Hz}$ means that the pumps are consuming higher power for low flow rate. In ESP optimization, the target is to produced higher fluid flow rates at least power consumption possible. ESP must be operated within the frequency of $45 \mathrm{~Hz}-80 \mathrm{~Hz}$. Operation of ESP outside this window reduces the operating life of the pump. In apportioning flow rates which is determined by ESP frequency and well chokes, it is important to consider the capacity of the separators and the surface storage in order to accommodate produced fluids especially in offshore location where space is a major limitation.

\section{Methodology}

The methods comprise formulating the equations for ESP, forming the optimization equations and constraints, gathering of data, modeling and simulation in Prosper and GAP software.

\subsection{Formulation of Equation for ESP}

ESP as a type of centrifugal pump and like other centrifugal pumps, ESP does not displace a fixed amount of liquid but it keeps its pressure relatively constant in the flow system. Thus the output flow rate depends on the backpressure.

The Pumping head for ESP is given as:

$$
h=\frac{\Delta p}{0.433}
$$




$$
\begin{gathered}
\Delta p=p_{\text {discharge }}-p_{\text {suction }} \\
p_{\text {suction }}=p_{\text {wf }}-0.433 \gamma_{L} D
\end{gathered}
$$

where: $h=$ pumping head, $\mathrm{ft}, \Delta p=$ Pressure differentials, psi;

$$
p_{\text {discharge }}=\text { discharge pressure, psi, } p_{\text {suction }}=\text { Suction pressure, psi; }
$$

$\gamma_{L}=$ Specific gravity of production fluid, $D=$ Depth of production interval, ft.

The minimum pump depth is given by:

$$
D_{\text {pump }}=D-\frac{p_{w f}-p_{\text {suction }}}{0.433 \gamma_{L}}
$$

where: $D_{\text {pump }}=$ Minimum pump depth, $\mathrm{ft}$.

The number of stage for ESP is given as:

$$
n_{s}=\frac{Z}{L_{s}}
$$

where: $n_{s}=$ number of design stages, $Z=$ Total dynamic head, $\mathrm{ft}, L_{s}=$ Lift per stage.

The equation for ESP motor horsepower calculation is given as:

$$
P_{h m}=P_{h s} \times n_{s} \times \rho_{f}
$$

where: $P_{h m}=$ motor horsepower; $P_{h s}=$ horsepower per stage; $n_{s}=$ number of design stages.

$\rho_{f}=$ specific gravity of fluid.

\subsection{Formulation of the Optimization Problem}

Consider a field where ESP is to be applied to a cluster of $N$ wells. The wells share common manifold, separator and flow lines, and are tied to each other. If we assume that the separator is close enough to the production manifold such that there is negligible pressure difference between separator and production manifold and that flow lines are horizontal. Then, the total oil production rate for the field $Q_{o}$ from all the $N$ wells is the sum of the individual well's oil production $q_{o i}$

Mathematically:

$$
Q_{o}=\sum_{i=1}^{N} q_{o i}
$$

\subsection{Gas Lift Optimization Problem}

Oil production maximization (objective function):

$$
Q_{o}=\max \sum_{i=1}^{N} q_{o i}
$$

Equation (8) is subject to the following constraints:

1) The ESP intake pressure $\left(P_{\text {in_ESP }}\right)$ should be equal to or greater than the bubble point pressure $\left(P_{\text {bub }}\right)$ to avoid cavitation:

$$
P_{\text {in_ESP }} \geq P_{\text {bub }}
$$

2) The flow rate of fluid within the pump must be within the operating win- 
dow. That is to say the fluid flow rate within the pump should not be greater than the flow rate that corresponds to the maximum frequency and should not be less than the flow rate that corresponds to the minimum frequency:

$$
Q_{\text {fr_min }} \leq q_{l} \leq Q_{m f r_{-} a x}
$$

3) The speed of the pump (frequency) should be between 50 and 70 for speed constraint 1 and 45 and 80 hertz for speed constraint 2:

$$
\begin{aligned}
& 50 \leq f_{r} \leq 70 \quad(\text { Speed constraint } 1) \\
& 45 \leq f_{r} \leq 80 \quad(\text { Speed constraint } 2)
\end{aligned}
$$

4) The production choke valve should be within 0 and 100:

$$
0 \leq v \leq 100
$$

5) The liquid produced should not be greater than the maximum capacity of the storage:

$$
\begin{array}{ll} 
& \sum_{i=1}^{N} q_{l i} \leq Q_{\operatorname{lmax}} \\
& Q_{\text {max }}=Q_{w}+Q_{o} \\
\text { (i) } & Q_{\text {max }} \leq 70000 \mathrm{stb} / \mathrm{d} \\
\text { (ii) } & \text { Watercut }(W C) \leq 0.8
\end{array}
$$

6) Separator pressure must not exceed 250 psig;

7) Node pressure must not exceed reservoir pressure.

\subsection{Sequential Quadratic Programming in GAP}

The natural responses of individual wells in a field to the well's production rate are given below using the nodal analyses. For each node the following relation can be observed:

$$
\begin{gathered}
\sum_{i=1}^{n} \dot{m}=0 \\
P_{\text {ups }}-P_{\text {down }}-\Delta P=0 \\
\Delta P=f(\dot{m}, p, T, L) \\
P_{\text {sep }}=\text { Constant }
\end{gathered}
$$

\subsection{Field Case Study}

Field AKUX is an onshore field. This field has two reservoirs and a total of five wells drilled to it. Well-1 and Well-2 are from reservoir AKUXR1 while Well-3, Well-4 and Well-5 are from reservoir AKUXR2. In the early life of the field, these wells were operated solely using the primary energy inherent in the reservoir (primary drive mechanism). It was later decided that artificial lift was used to complement the natural production. Electrical submersible pump was selected after rigorous section criteria performed. Because of the peculiarity of each well in its geometry and completion, ESP performance on each well varied in the field. Each well has its own optimum production potentials if the wells are treated as separate units. This is done in well optimization, where the optimization is focused at 
well level. In the field, the network of wells imposed further constraints the system when optimization is required. Because the wells are linked up to the field network system, production activities on one well affects the other. To fully optimize the field, it is necessary to consider at the field level, the conditions necessary for optimum oil production in the field given certain constraints imposed by pump frequency, separator size and pressure, etc. In this work, the following are considered: 1) Five wells in a field of two reservoirs are completed with Electrical submersible pumps. Each pump in a well is controlled by the Variable Speed Drive (VSD) unit that is installed near the well; 2) Surface network manifold, flow lines and pipelines help to gather production from each well and take it to a common processing facility.

\subsection{Modeling}

Advanced production and system performance analyses software (PROSPER) and general Allocation Package (GAP) software was used for modeling in this research work. Prosper was used to model well-by well ESP design and performance while GAP was used to model the field network optimization performance. Figure 1 shows the modeling workflow while Table 1 shows the data utilized for the modeling.

PVT data and then adjusting the correlation model until there is fit with the measured data. The potentials of the well and pressure loss calculations during production depends highly on the accuracy of the PVT data. The IPR and VLP curves were generated for each well. In matching the VLP, non-linear regression techniques were utilized by prosper to optimize the various components entered for the optimization. The matching of the VLP helps to reveal the inconsistencies in the PVT model or in the equipment description which were adjusted for accurate results.

\subsubsection{Construction of the Well Model}

All the wells were modeled individually in prosper. PVT data for each well were entered and regressed to match standard correlations. Each well's data includes deviation surveys, downhole completion, geothermal gradient and average heat capacities. ESP properties for each well in well-by-well ESP includes, pumps selection, motors selection and turbine selection. In prosper modeling, the downhole-equipment gradient, inflow performance and ESP designs were modeled for

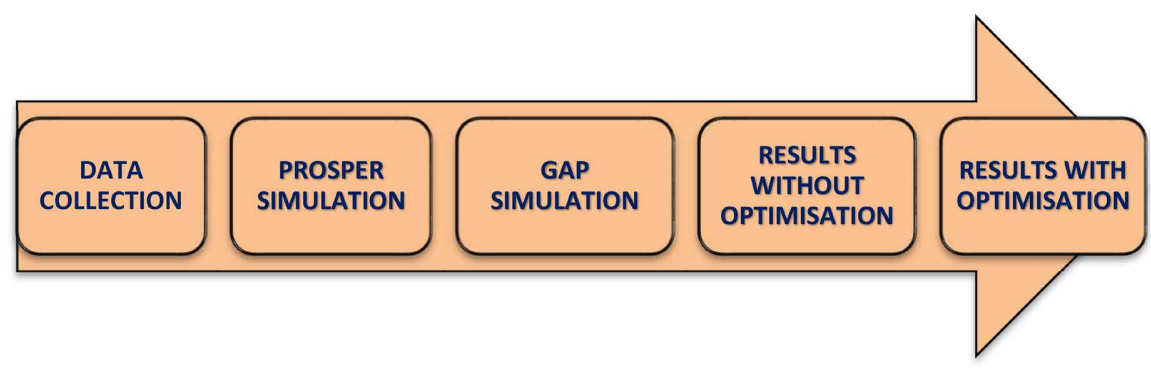

Figure 1. Workflow. 
Table 1. Reservoir, well and PVT data utilized for the study.

\begin{tabular}{|c|c|c|c|c|c|}
\hline Parameter & Well 1 & Well 2 & Well 3 & Well 4 & Well 5 \\
\hline Reservoir pressure (psi) & 4490 & 4490 & 4458 & 4458 & 4458 \\
\hline Bubble point pressure (psi) & 1958 & 1958 & 1940 & 1940 & 1940 \\
\hline Reservoir temperature $\left({ }^{\circ} \mathrm{F}\right)$ & 210 & 210 & 210 & 210 & 210 \\
\hline Reservoir permeability $(\mathrm{mD})$ & $600-800$ & $600-800$ & $600-800$ & $600-800$ & $600-800$ \\
\hline Reservoir thickness (ft) & 100 & 100 & 120 & 120 & 120 \\
\hline Average porosity & 0.20 & 0.20 & 0.25 & 0.25 & 0.25 \\
\hline Solution GOR (scf/stb) & 507 & 507 & 434 & 434 & 434 \\
\hline Oil gravity (API) & 34.2 & 34.2 & 31 & 31 & 31 \\
\hline Oil viscosity (cP) & 0.63 & 0.63 & 1.1 & 1.1 & 1.1 \\
\hline Gas Gravity (SG) & 0.92 & 0.92 & 0.91 & 0.91 & 0.91 \\
\hline Water salinity (ppm) & 220,000 & 220,000 & 220,000 & 220,000 & 220,000 \\
\hline Well depth (ft) & 11,188 & 11,200 & 11,244 & 10,748 & 11,200 \\
\hline Tubing size (inch) & 3.5 & 4.5 & 4.5 & 4.5 & 3.5 \\
\hline Geothermal gradient $\left(\mathrm{BTU} / \mathrm{h} / \mathrm{ft}^{2} /{ }^{\circ} \mathrm{F}\right.$ & 8.383 & 8.43 & 8.4 & 8.71 & 10.16 \\
\hline Watercut (\%) & 16 & 60 & 40 & 10 & 30 \\
\hline PI (stb/d/psi) & 13 & 3.8 & 4.7 & 14.7 & 10 \\
\hline Oil fvf (rb/stb) & 1.35 & 1.35 & 1.29 & 1.29 & 1.29 \\
\hline
\end{tabular}

each well. VLP curves were generated for varying water cuts, GOR, skin, node pressures, and ESP motor speeds and exported to GAP (the surface network simulator). Figure 2 depicts ESP pump plot for the well operation.

\subsubsection{Field Network Modelling}

The field network model was achieved using GAP software. GAP is a multiphase system optimization software that models and optimizes both production and injection networks. The network here is the general network consisting of both surface and downhole components. In its optimization method, GAP uses a nonlinear sequential quadratic programming technique. GAP allows to maximization of certain objective functions while honoring certain constraints imposed in the system. In simulating the field network models, the VLP curves generated in Prosper for each well is exported to GAP. Upon running the GAP model for the field network model, both the IPR and VLP curves were extracted.

\subsubsection{Construction of the Surface Network of the Field}

GAP software was used to model the surface network system. The surface network system comprises the ESP production systems for the well clusters. The ESP production system consists of the wells ( 5 wells), wellheads, flow lines, manifolds and separator. There are two types of flow lines here: one is the flow lines 


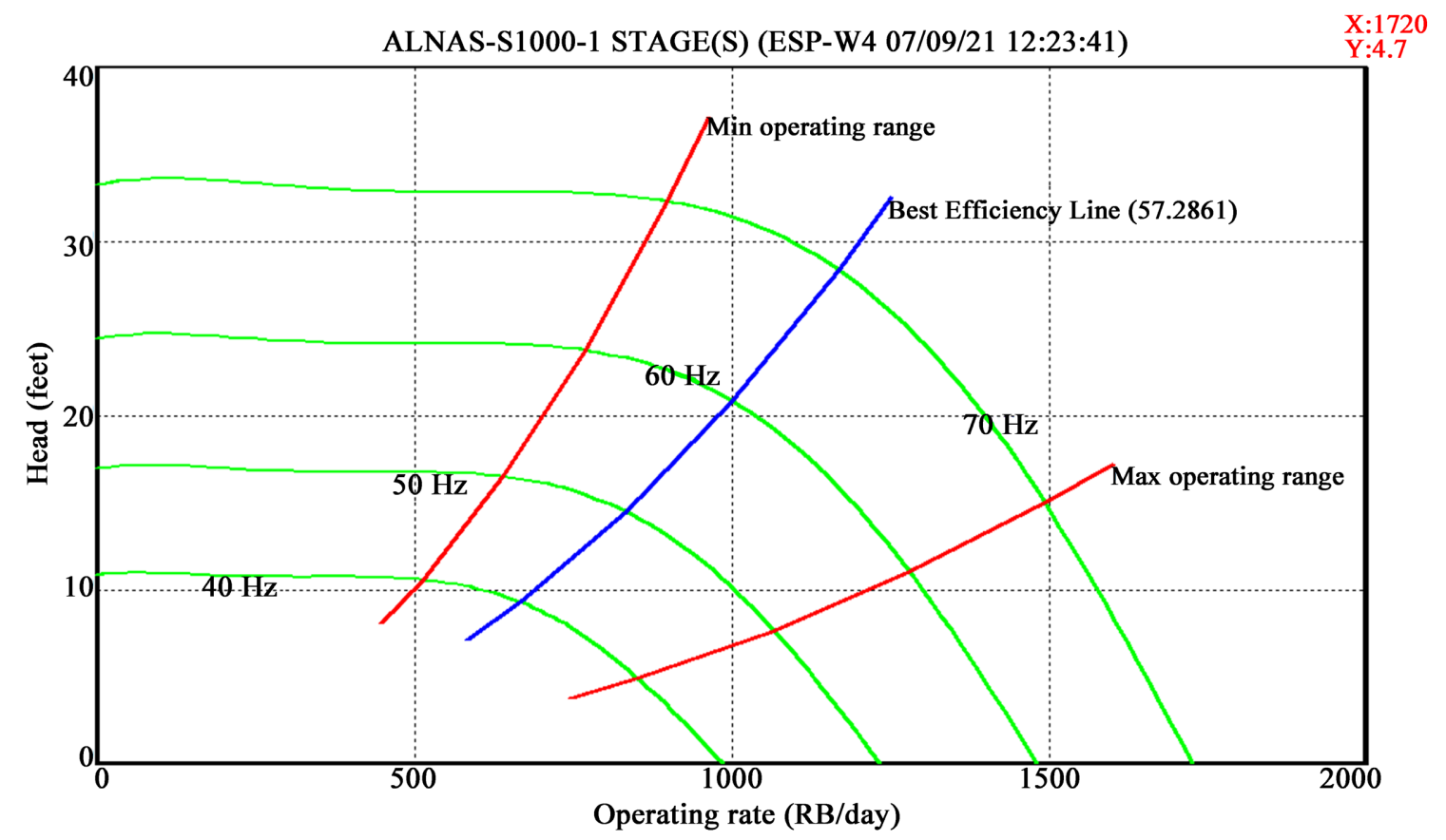

Figure 2. ESP pump plot for well operation.

from wellheads to the manifolds and the other is the flow lines from the manifolds to the low pressure single stage separator. The pipelines used were properly defined. In the definition, their lengths, inner diameters, roughness and multiphase correlations were inputted and used to calculate the pressure drops. The pipelines were then matched with the standard pipeline correlations chosen. The field network model was simulated for two cases: Case 1 is running the GAP simulation without optimization. Here, the required ESP frequency for each well was inputted and GAP calculates the individual productions from each well and also the total production for the whole field which is a function of the reservoirs, the wells in the field and the motor frequency. For these, three ESP frequencies were considered. They are $50 \mathrm{~Hz}, 60 \mathrm{~Hz}$ and $70 \mathrm{~Hz} .60 \mathrm{~Hz}$ was taken as the base frequency. Case 2 is running the GAP simulation with optimization. In the GAP optimization case, ESP frequencies are not entered as input but GAP software is allowed to run by performing optimization and determining the best ESP frequencies to achieve optimum field liquid production rate for the field. Thus, the ESPs shown after the optimization solver finishes the network solution because the optimum ESP frequency to achieve optimum production rates for the field. In achieving the optimization case, constraints were imposed on the ESP pump system. The first constraint on the frequency of the ESP pump is that the frequency must fall between $50 \mathrm{~Hz}$ and $70 \mathrm{~Hz}$. The second constraint imposed on the ESP pump is that the frequency of the ESP pump must fall between $45 \mathrm{~Hz}$ and $80 \mathrm{~Hz}$. Other constraints imposed on the field network system are outlined in section 3.3. The target here is not on well basis but the entire field network with cluster of five wells communicating together. Figure 3 depicts the ESP System Field Production Network. 


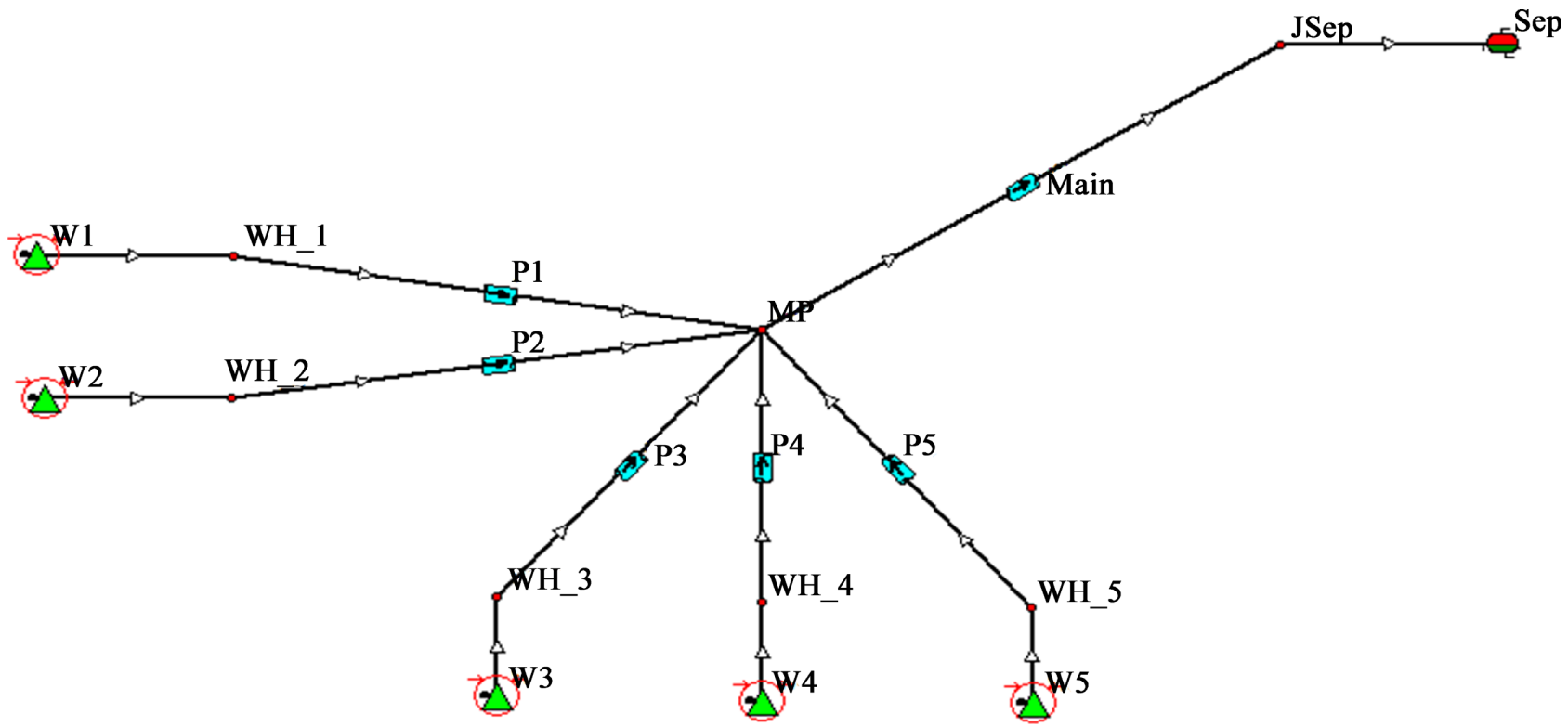

Figure 3. ESP system field production network.

\section{Results and Discussions}

The results of the simulations performed in Prosper and GAP are given and discussed in this section. The results are given for the simulation without optimization and with optimization for the field network modeling.

\subsection{Case 1: Simulation without Optimization}

In simulating for case 1, GAP software was modeled by entering a fix value for the ESP frequency with $60 \mathrm{~Hz}$ as base frequency and subsequently sensitivity of $50 \mathrm{~Hz}$ and $70 \mathrm{~Hz}$ were analyzed. For the base frequency, 200 psig was considered as the separator pressure. Separator pressure relates to the pressure of the separation process. In the sensitivity analyses, effects of separator pressure on production rates and recovery were analyzed. Separator pressures of 180 psig, 140 psig, 120 psig, 100 psig and 80 psig were further considered alongside that of 200 psig taken as base. The results are given for well-by-well simulation basis and for field network simulation basis. The well-by-well simulation results gives the production rates corresponding to the ESP input parameters for that particular well. Being that each well differ in well properties such as deviation, drilling and completion design, ESP design features; the production rates from individual wells will be different as production from wells are a non-linear of different contributory factors prevalent in the well and the reservoir. On the other hand, for the field base simulation without optimization, the field was considered to be a network of five wells communicating with each other. However, because the GAP stimulation was not run with optimization in this case, individual well frequencies were entered as input variables in the GAP software and the software was allowed to calculate the production rates for the field given the frequencies entered for the wells in the field. 


\subsubsection{Well-by-Well Production Rates at $60 \mathrm{~Hz}$ and 200 Psig Separator Pressure}

The production result for well-by-well simulation using $60 \mathrm{~Hz}$ ESP frequency and 200 psig separator pressure is given in Figure 4. From Figure 4, it can be observed that well 4 has the highest oil rate followed by well 1 . The well with the least oil rate is well 2. Similarly, well 4 has the highest liquid rate followed by well 1 while well 1 has the least liquid rate. In analyzing the water rate, well 2 has the highest water rate followed by well 3 . The well with lowest water rate is well 4 . The individual well production capacity reveals their capacity to be lifted by the electrical submersible pump. Well 4 has the best performance than all the other wells with low water production and higher oil production than all the other wells. Since all the wells were produced with same operating ESP pressures it can be observed that well 2 produces more water than oil, owing to this ESP pump would be the best choice to produce well 2 because of the high water cut. For all the wells, the water cut constraints of not more than $80 \%$ was met. The highest water cut was for well 2 with $40 \%$ water cut. The tabular display of the simulation result for well-by-well simulation using ESP of $60 \mathrm{~Hz}$ at 200 psig separator pressure is given in appendix.

\subsubsection{Field Performance at Varied Separator Pressures for Base ESP Frequency $(60 \mathrm{~Hz})$}

It was necessary to investigate the effect of separator pressure on the performance of the ESP system on the field network modeling. This was done by varying the separator pressure and observing changes in production rates and pump characteristic factors. Table 2 gives the results of the simulation conducted for several separator pressures on the base ESP frequency $(60 \mathrm{~Hz})$ while Figure 5 depicts the field oil rate for several separator pressures for the same base ESP frequency. It is evident from Table 2 that the lower the pressure of separation, the higher the rates of production and also the pump power requirement and the surface voltage. But the degree of this change is important. A change from separator pressure of 200 psig to 80 psig gives an increased field oil rate of 547 $\mathrm{stb} / \mathrm{d}$. representing $1.69 \%$. Decreasing the separator pressure creates higher pressure differentials and thus higher driving energy for the fluids to be produced.

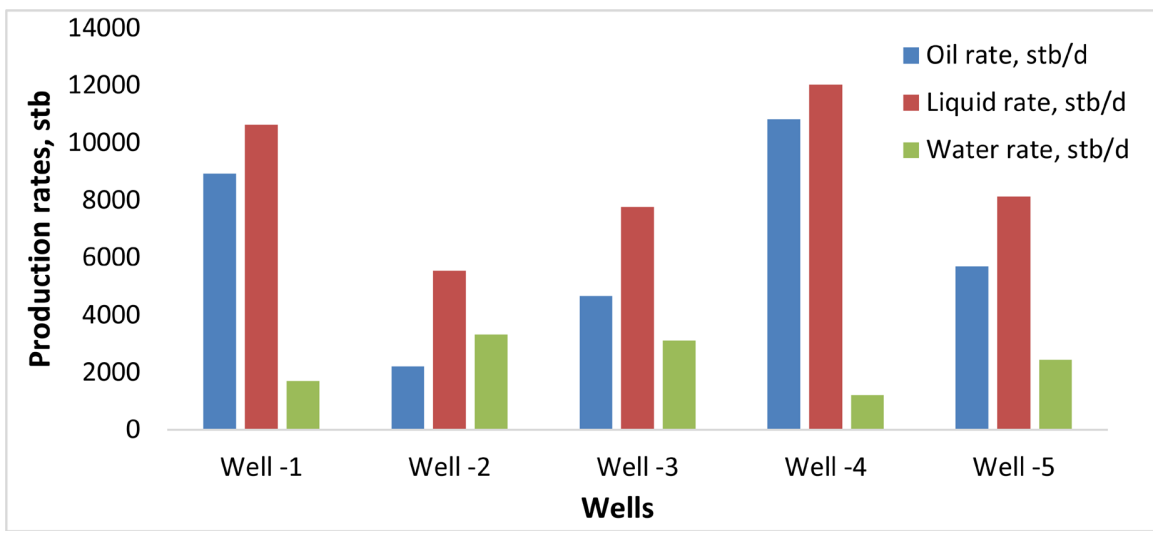

Figure 4. Well-by-well results of production rates for case 1 (without optimization). 
Table 2. Field performance without optimization at varied separator pressure for the base case.

\begin{tabular}{|c|c|c|c|c|c|c|}
\hline $\begin{array}{c}\text { Separator } \\
\text { pressure }\end{array}$ & $\begin{array}{l}\text { Oil rate } \\
\text { stb/d }\end{array}$ & $\begin{array}{l}\text { quid rate } \\
\text { stb/d }\end{array}$ & $\begin{array}{c}\text { Vater rat } \\
\text { stb/d }\end{array}$ & $\begin{array}{l}\text { Gas rate, } \\
\text { MMscf/d }\end{array}$ & $\begin{array}{c}\text { Imp powe } \\
\mathrm{hp}\end{array}$ & $\begin{array}{l}\text { tace volt } \\
\text { volts }\end{array}$ \\
\hline 200 & 32,320 & 35,970 & 11,780 & 15.10 & 1752.6 & 3172.91 \\
\hline 180 & 32,430 & 361,220 & 11,830 & 15.16 & 1753.7 & 3174.02 \\
\hline 160 & 32,540 & 36,260 & 11,870 & 15.20 & 1754.78 & 3175.12 \\
\hline 140 & 32,640 & 36,390 & 11,920 & 15.25 & 1755.73 & 3176.1 \\
\hline 120 & 32,730 & 36,500 & 11,960 & 15.29 & 1756.59 & 3176.99 \\
\hline 100 & 32,800 & 36,600 & 11,990 & 15.32 & 1757.34 & 3177.76 \\
\hline 80 & 32,870 & 36,680 & 12,020 & 15.34 & 1757.97 & 3178.38 \\
\hline
\end{tabular}

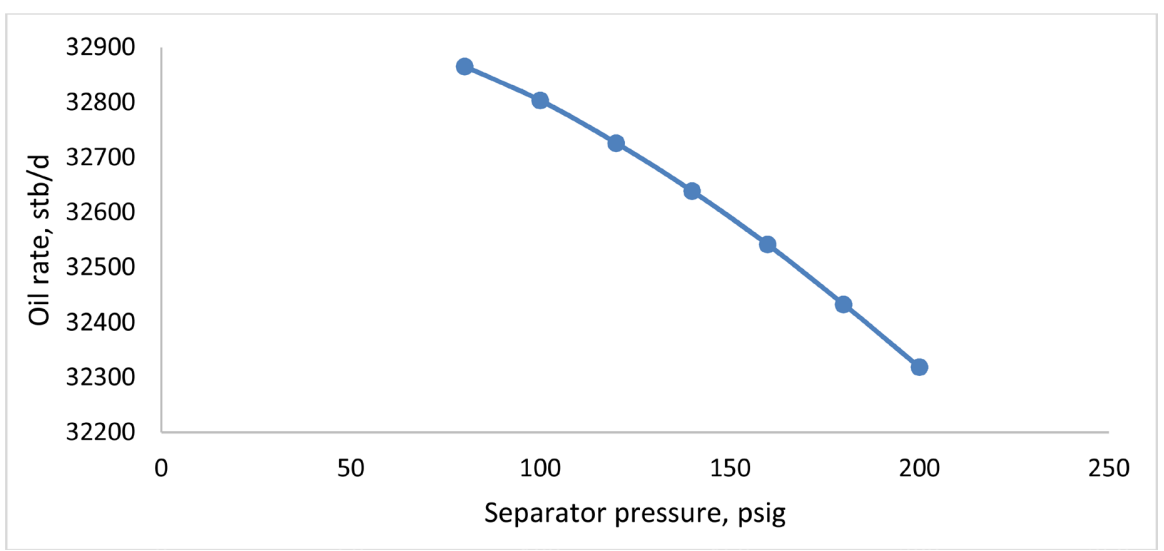

Figure 5. Oil rate as a function of separator pressures at $60 \mathrm{~Hz}$ ESP motor frequency.

Since the reservoir and wellhead pressures are not altered but kept constant, reducing the separator pressure creates higher energy of flow for the fluids from the reservoir to the stock tank. However reducing the separator pressure creates more favourable environment for more gases to be produced as is evident in $\mathrm{Ta}$ ble 2. A reduction in pressure from 200 psig to 80 psig leads to an additional gas production of $0.24 \mathrm{MMscfd}$ representing an increase in gas production of $1.6 \%$. Additionally, beaming up (i.e., in this case reducing separator pressure) has the additional consequence of leading to water coning or gas cupping which would be an operational difficulty and costly to handle. Since the separator pressure is part of the integrated production system which starts from the reservoir down to the separator, the optimum separator pressure would be based on the liquid and gas handling capabilities of the field. Although decreasing the separator pressure increases the production rate, but the increase is not profound as can be observed from Table 2. From Figure 5, it can be observed that the oil rate increases with decrease in separator pressure (from 200 psig to $80 \mathrm{psig}$ ). This increase is attributed to higher pressure differentials created in the production system. Since the field network system is modeled as a complete single integrated system from the well to the surface separator, decreasing the pressure at the separator 
creates higher energy of low for the wellbore fluids to come to the surface. This however includes increase e in both the gas production rates and the water production rates which may create additional gas and water handling problems since there is a constraint on the surface liquid and gas handling volumes.

\subsubsection{Field Performance at Different Frequencies and Separator Pressures} The oil rate for different frequencies and separator pressures for case 1 (without optimization) is depicted in Figure 6. From Figure 6, it can be observed that the higher the ESP motor frequency, the higher the oil rate. A decrease from the base frequency of $60 \mathrm{~Hz}$ to $50 \mathrm{~Hz}$ decreased the oil rate by $13.85 \%$ while an increase in ESP motor frequency from $60 \mathrm{~Hz}$ to $70 \mathrm{~Hz}$ increased the oil rate by $15 \%$. Similarly, the liquid rate for varied ESP motor frequency is depicted in Figure 7. The field liquid rate for $60 \mathrm{~Hz}$ ESP motor frequency is 44,090 stb/d while the liquid rate for $50 \mathrm{~Hz}$ and $70 \mathrm{~Hz}$ ESP motor frequencies are 37,540 stb/d and 51,150 $\mathrm{stb} / \mathrm{d}$ respectively. A decrease from $60 \mathrm{~Hz}$ to $50 \mathrm{~Hz}$ reduced the field liquid rate by $14.87 \%$ while an increase from $60 \mathrm{~Hz}$ to $70 \mathrm{~Hz}$ increased the field liquid rate by $16 \%$.

\subsection{Case 2: Simulations with Optimization}

For the field network simulation with optimization, network analysis was performed

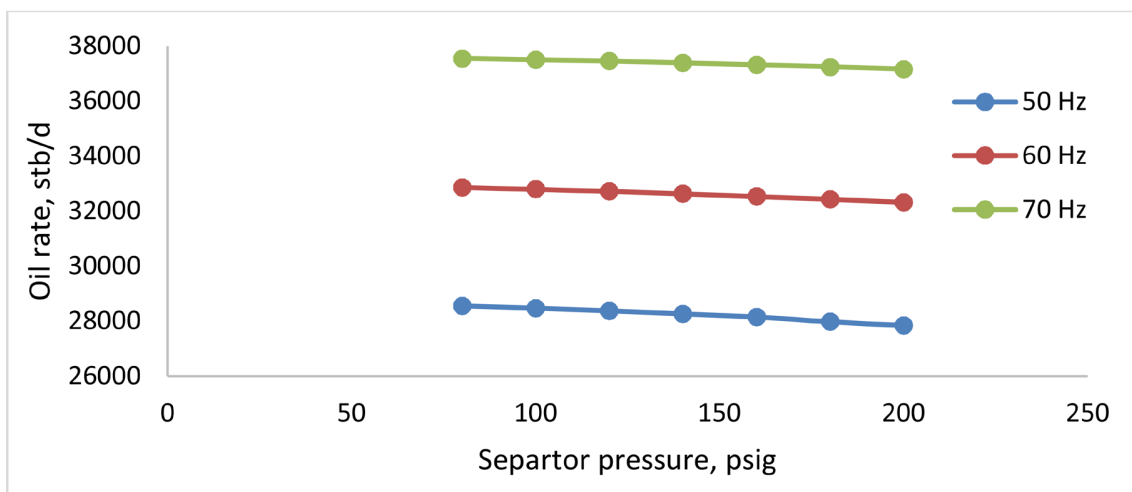

Figure 6. Oil rate for different ESP motor frequency at base separator pressure of 200 psig.

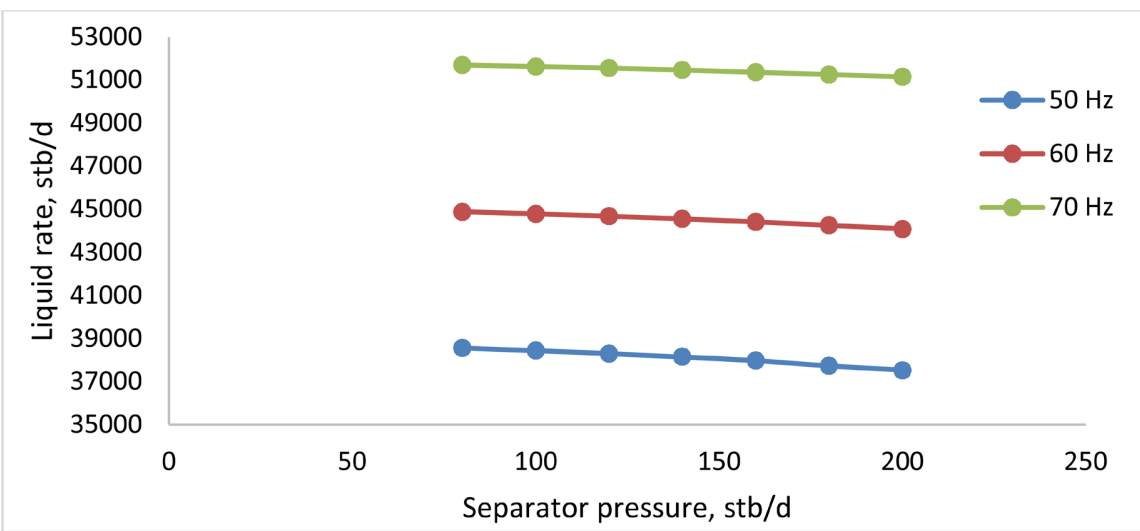

Figure 7. Liquid rate for different ESP motor frequency at base separator pressure of 200 psig. 
in GAP with the "optimize all constraints" checked. This simulation technique allows the software to determine the conditions that will yield the optimum production rates while honouring the imposed constraints in the field and on the ESP system. GAP software was allowed to calculate by optimization technique the best ESP motor for each well that will lead to optimum total oil production for the field. Two types of constraints based on the frequency limits were observed. The first constraint on the motor frequency is that the frequency of each well should not be less than $50 \mathrm{~Hz}$ and should not be greater than $70 \mathrm{~Hz}$. The second ESP motor frequency constraint considered is that the motor frequency should not be less than $45 \mathrm{~Hz}$ and should exceed $80 \mathrm{~Hz}$. These two constraints on motor frequency are evaluated to determine the effect of lower and upper boundaries of motor frequencies on the performance of the ESP system in field network optimization of oil wells.

\subsubsection{Oil Production Rate for Case 2}

Table 3 depicts ESP simulation with optimization for case 2 while Figure 8

Table 3. ESP simulation with optimization for case 2 for different fluid rates.

\begin{tabular}{ccccccc}
\hline \multirow{2}{*}{$\begin{array}{c}\text { Separator } \\
\text { pressure, } \\
\text { psig }\end{array}$} & \multicolumn{2}{c}{ Oil rate, stb/d } & \multicolumn{2}{c}{ Liquid rate, stb/d } & \multicolumn{2}{c}{ Water rate, stb/d } \\
\cline { 2 - 6 } & $\begin{array}{c}\text { Constraint } \\
\text { stb/d }\end{array}$ & $\begin{array}{c}\text { Constraint } \\
\text { stb/d }\end{array}$ & $\begin{array}{c}\text { Constraint } \\
\text { stb/d }\end{array}$ & $\begin{array}{c}\text { Constraint } \\
\text { stb/d }\end{array}$ & $\begin{array}{c}\text { Constraint } 1, \\
\text { stb/d }\end{array}$ & $\begin{array}{c}\text { Constraint 2, } \\
\text { stb/d }\end{array}$ \\
\hline & 32,690 & 33,180 & 43,710 & 43,650 & 11,020 & 10,470 \\
180 & 32,790 & 33,290 & 43,850 & 43,760 & 11,060 & 10,470 \\
160 & 32,890 & 33,390 & 44,000 & 43,890 & 11,110 & 10,500 \\
140 & 32,980 & 33,480 & 44,130 & 44,030 & 11,140 & 10,550 \\
120 & 33,060 & 33,560 & 44,250 & 44,160 & 11,190 & 10,600 \\
100 & 33,130 & 33,630 & 44,340 & 44,250 & 11,210 & 10,620 \\
80 & 33,190 & 33,680 & 44,420 & 44,330 & 11,230 & 10,660 \\
\hline
\end{tabular}

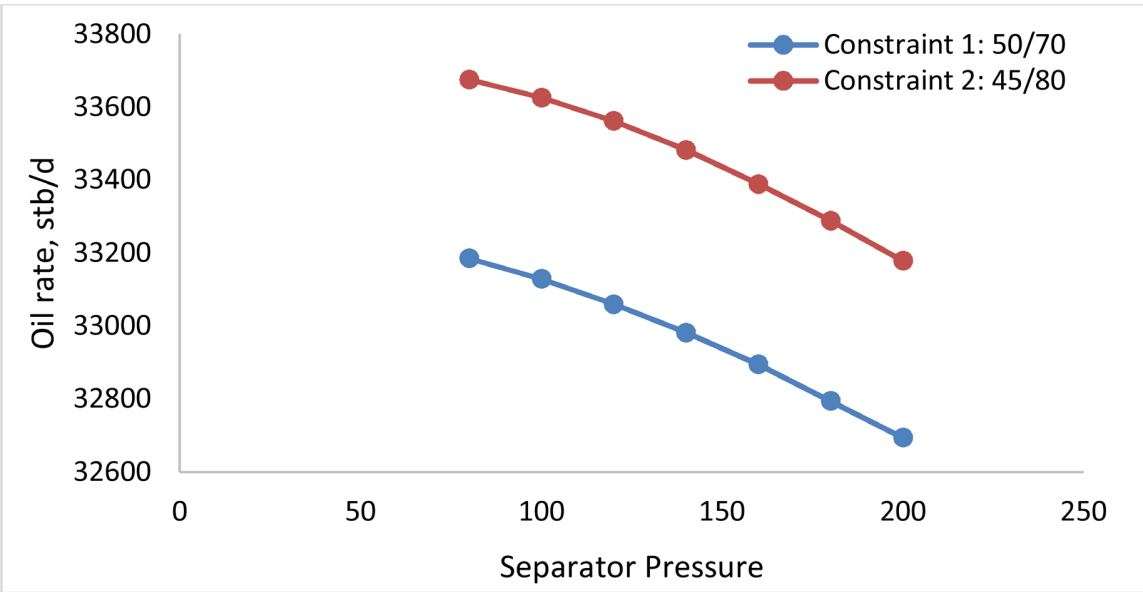

Figure 8. Oil rate vs Separator pressure for ESP simulation with optimization at different constraints. 
depicts Oil rate as a Separator pressure function for ESP simulation with optimization at different constraints. From Table 3, the oil rate at 200 psig was calculated to be $32,694.26 \mathrm{stb} / \mathrm{d}$ for constraint $1(50 \mathrm{~Hz} / 70 \mathrm{~Hz})$ and 33,180 stb/d for constraint $2(45 \mathrm{~Hz} / 80 \mathrm{~Hz})$. Also from Figure 8, it can be observed that constraint 2 had higher field oil rate than constraint 1 . This is attributed to higher frequency interval of $35 \mathrm{~Hz}(45 \mathrm{~Hz} / 80 \mathrm{~Hz})$ for constraint 1 as against $20 \mathrm{~Hz}(45 \mathrm{~Hz} / 80 \mathrm{~Hz})$ for constraint 1 . The liquid rate for constraint 1 and 2 are 43,720 stb/d and 43,650 $\mathrm{stb} / \mathrm{d}$ respectively. There were more production rates for constraint 2 than 1 and this again is attributable to higher frequency interval. Constraint 2 had an additional oil rate of $485 \mathrm{stb} / \mathrm{d}$ than constraint 1 . Thus more oil was produced from constraint 2 . Nevertheless, more liquid was produced from constraint 1 . The liquid rate of constraint 1 surpassed that of constraint 2 by $68 \mathrm{stb} / \mathrm{d}$. From constraint 1 , the water produced was $11,020 \mathrm{stb} / \mathrm{d}$ while the water rate for constraint 2 was $10,470 \mathrm{stb} / \mathrm{d}$. Thus more water was produced for constraint 1 than for constraint 2 and this attributed to the higher liquid rate observed in Table 3.

Figure 9 shows the oil production for individual wells for ESP optimization with two ESP constraints imposed for case 2. It can be observed from Figure 9 that the difference between the oil rate for constraint 1 and constraint 2 is very small for the other wells except for well 4 . The percentage difference in oil rate for constraint 1 and 2 are given as: $-2.32 \%$ for well $1,-19.27 \%$ for well $2,-3.54 \%$ for well 3, 11.15\% for well 4 and $-3.11 \%$ for well 5 . As can be observed, for all the five wells, constraint 1 gave higher oil rate than constraint 2 except for well 4 . Well 4 has a positive percentage difference which means that constraint 2 has greater oil rate than constraint 1 . This higher oil rate for well 4 of constraint 2 , entails higher pump power requirement for well 4 . The oil rate for well 4 greatly surpasses those of the other wells. Even though for well 1, well 2, well 3 and well 5 the oil rate for constraint 1 is higher than that of constraint 2 , the total oil rate for constraint 2 is higher than that of constraint 1 because of the high oil rate from well 4 which offsets the production rate from the other wells and makes constraint 2 to have higher total oil production rate.

\subsubsection{ESP Pump Power}

The pump power is the horse power requirement for each ESP pump operating

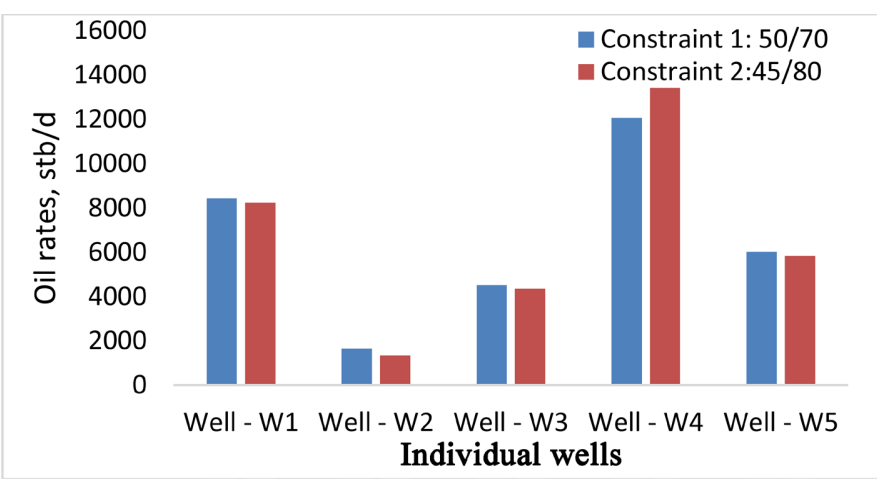

Figure 9. Oil rate for individual wells for ESP optimization with constraints. 
in each well. Depicted in Figure 10 is the Pump power for individual wells for ESP optimization with constraints. From Figure 10, it can be observed that for all the wells aside well 4, constraint 1 requires higher pump power operation of the wells than constraint 2 . Thus, constraint 2 is more conservative in energy usage than constraint 1 for well 1 , well 2, well 3 and well 5 . Nevertheless, well 4 prove otherwise with constraint 2 requiring higher pump power than constraint 1. More pump power was required for well 4 of constraint 2 because well 4 yields higher oil rate than other wells of constraint 2 as can be observed in Figure 9. However, more pump power signifies more energy requirement and thus more operational expenditure. Thus, the incremental oil rate must be able to offset the operational cost in terms of energy requirement (i.e., electrical power requirement to run the ESP) before the conclusion is made as to the constraint that is most conservative.

\subsection{Comparison of Simulation with Optimization and without Optimization}

Figure 11 depicts comparison of Oil rates for ESP simulation with and without optimization while Table 4 depicts Pump efficiencies of the ESP pumps in different applications in the field. It is important to determine the incremental oil rate accruing to the two cases considered in order to suggest the best approach for optimum recovery of oil in field of clustered wells. From Figure 11, it can be observed for ESP frequency values of $50 \mathrm{~Hz}$ and $60 \mathrm{~Hz}$ that performing field network with optimization produces more oil than performing field network without optimization. This is because for the case of optimization, the GAP solver calculates between ESP frequencies of $50 \mathrm{~Hz}$ and $60 \mathrm{~Hz}$, the best ESP frequency that would yield the optimum production rate for the field. This is calculated based on the inherent intrinsic properties of each well and for the field as a whole when the wells are connected together. By this, the effect of backpressures from the communicating wells and lines at the surface have been accommodated and the best ESP frequency requirements that would cause the least resistance to cumulative fluid flow from the well cluster are given as the calculated

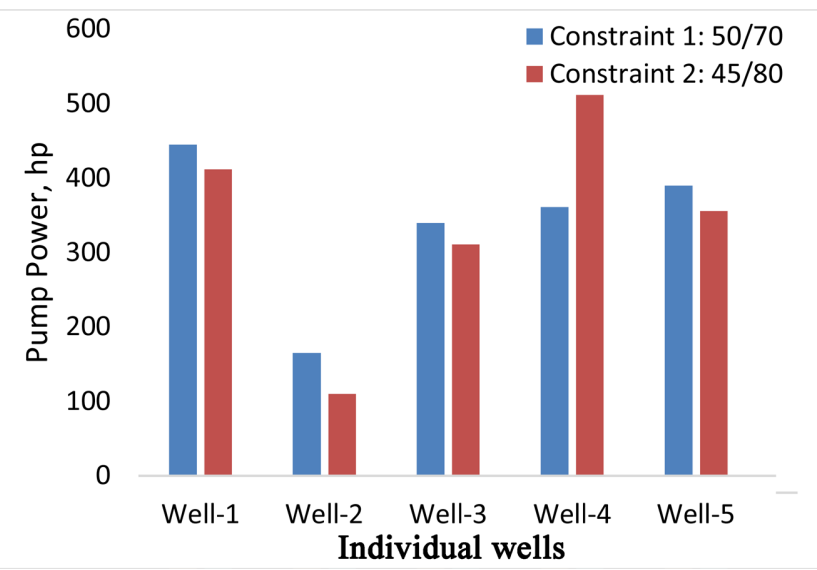

Figure 10. Pump power for individual wells for ESP optimization with constraints. 
Table 4. Pump efficiencies of the ESPs in different applications in the field.

\begin{tabular}{cccccc}
\hline \multirow{2}{*}{ Wells } & \multicolumn{2}{c}{ Case 2: with optimization } & \multicolumn{3}{c}{ Case 1: without optimization } \\
\cline { 2 - 6 } & \multicolumn{2}{c}{ Pump efficiency } & \multicolumn{3}{c}{ Pump efficiency } \\
\cline { 2 - 6 } & Constraint 1 Constraint 2 & $50 \mathrm{~Hz}$ & $60 \mathrm{~Hz}$ & $70 \mathrm{~Hz}$ \\
\hline Well-W1 & 0.64 & 0.64 & 0.64 & 0.64 & 0.64 \\
Well-W2 & 0.64 & 0.62 & 0.64 & 0.64 & 0.64 \\
Well-W3 & 0.68 & 0.69 & 0.69 & 0.69 & 0.67 \\
Well-W4 & 0.65 & 0.69 & 0.64 & 0.65 & 0.65 \\
Well-W5 & 0.64 & 0.64 & 0.63 & 0.65 & 0.64 \\
Average & 0.65 & 0.656 & 0.648 & 0.654 & 0.648 \\
\hline
\end{tabular}

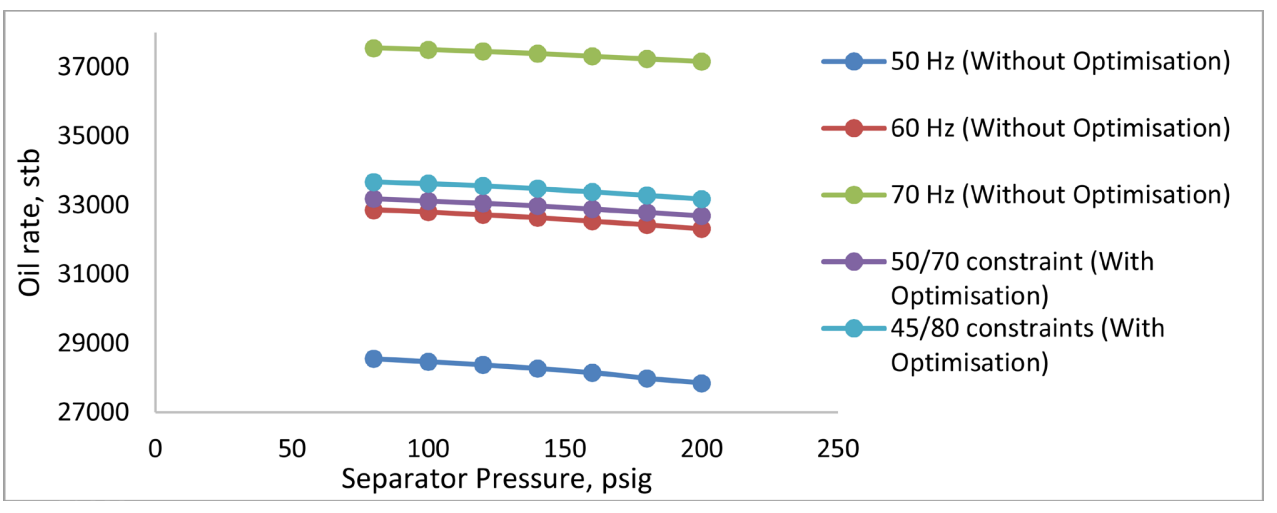

Figure 11. Oil rates for ESP simulation with and without optimization.

ESP frequencies corresponding to the ESP frequency constraints imposed (in this case between $50 \mathrm{~Hz}$ and $70 \mathrm{~Hz}$ ). Conversely, with ESP frequency of $70 \mathrm{~Hz}$ for case 1, the production rate exceeded that of case 2. This is because for case 1 at ESP frequency of $70 \mathrm{~Hz}$, all the five wells operated at the $70 \mathrm{~Hz}$ ESP frequency. But for case 2, only well 4 operated at ESP frequency of $70 \mathrm{~Hz}$ as stated in Figure 9. Other wells operated at ESP frequencies below $70 \mathrm{~Hz}$ which affected their production rates because the production rate increases with increase in ESP frequency. In reality, ESP frequency is kept around $60 \mathrm{~Hz}$. If $60 \mathrm{~Hz}$ is taken as base, then it is justified that simulation with optimization yields higher oil rate than simulation without optimization. Thus, simulation with optimization as against simulation without optimization leads to increase in oil production rate of $1.16 \%$ for constraint 1 and $2.66 \%$ for constraint 2 respectively.

Thus, the field network optimization has enabled increased production of oil from the field resulting from recovery of different wells using electrical submersible pumps. The increased oil recovery in the field network analysis results from the use of modeling and simulation runs to aid decision making in field network analyses. The simulation runs pays off with increased recovery and revenue generation to the asset owner which ultimately leads to higher overall recovery efficiency from the field and increased motor and pumps efficiency in the field with the 
pump efficiencies given in Table 4. From Table 4, ESP simulation with constraint 2 gave the highest average pump efficiency. The average pump efficiency is the median of the pump efficiency of the individual well's ESP pump. The least pump efficiency was realized for ESP simulation without optimization at motor frequencies of $50 \mathrm{~Hz}$ and $70 \mathrm{~Hz}$. Depicted in Table 5 is the motor efficiency of the ESPs utilized for the simulation. From Table 5, it can be observed that simulations with optimization for constraint 2 had the highest motor efficiency of $87.5 \%$ followed by constraint 1 with motor efficiency of $87 \%$ than those of simulations without optimization with $84.8 \%, 85.4 \% 84.4 \%$ for $50 \mathrm{~Hz}, 60 \mathrm{~Hz}$ and $70 \mathrm{~Hz}$ respectively. The high efficiencies of the motors for constraints 2 and 1 also contributed to higher oil production rate of constraint 2 as depicted in Figure 9 followed by that of constraint 1 for case 2 with optimization. On the other hand, the low efficiencies of the motors for case 1 which were operated without optimization also contributed to lower oil production rate obtained at $50 \mathrm{~Hz}, 60 \mathrm{~Hz}$ and $70 \mathrm{~Hz}$.

Table 5. Motor efficiencies of the ESP pumps in several applications in the field.

\begin{tabular}{cccccc}
\hline \multirow{2}{*}{ Wells } & \multicolumn{2}{c}{ Case 2: with optimization } & \multicolumn{2}{c}{ Case 1: without optimization } \\
\cline { 2 - 6 } & \multicolumn{2}{c}{ Pump efficiency } & \multicolumn{3}{c}{ Pump efficiency } \\
\cline { 2 - 6 } & Constraint 1 & Constraint 2 & $50 \mathrm{~Hz}$ & $60 \mathrm{~Hz}$ & $\mathbf{7 0 ~ H z}$ \\
\hline Well-W1 & 0.88 & 0.88 & 0.87 & 0.88 & 0.85 \\
Well-W2 & 0.84 & 0.86 & 0.83 & 0.84 & 0.81 \\
Well-W3 & 0.88 & 0.88 & 0.88 & 0.88 & 0.85 \\
Well-W4 & 0.9 & 0.9 & 0.83 & 0.83 & 0.9 \\
Well-W5 & 0.85 & 0.854 & 0.83 & 0.84 & 0.81 \\
Average & 0.870 & 0.875 & 0.848 & 0.854 & 0.844 \\
\hline
\end{tabular}

\section{Conclusions}

Oil field network analyses have been conducted on the Niger Delta field AKUX comparing two reservoirs and five wells. An electrical submersible pump system has been deployed in the field to aid in oil production. It was required to determine the best ESP operability that will guarantee the highest oil recovery from the field. ESP motor frequency and separator pressure were selected as the variables on which sensitivities were performed. ESP simulations were done with and without optimization techniques using GAP software.

The following conclusions were drawn from this study:

1) Constraint 2 (comprising of $45 \mathrm{~Hz} / 80 \mathrm{~Hz}$, lower and upper frequency limits, respectively) yielded a higher oil rate than constraint 1 (comprising of 50 $\mathrm{Hz} / 70 \mathrm{~Hz}$, lower and upper frequency limits, respectively);

2) The average frequency for simulation with optimization (case 2) is $59.65 \mathrm{~Hz}$ for both constraint 1 and constraint 2; 
3) There was an increase in oil rate of $1.16 \%$ and $2.66 \%$ for constraints 1 and 2 when ESP simulation was carried out with optimization than without optimization;

4) The highest pump efficiency was realized for case 2 , constraint 2 . This justifies the better pump performance can be obtained in simulating with optimization;

5) Higher motor efficiencies were realized for case 2 (simulation with optimization) than for case 1 ;

6) It is recommended that simulation should be carried out to determine the optimum motor frequency for each well in the field of many wells. This frequency will yield the maximum field production recovery from the field and lead to higher revenues.

\section{Conflicts of Interest}

The authors declare no conflicts of interest regarding the publication of this paper.

\section{References}

[1] Epelle, E.I. and Gerogiorgis, D.I. (2017) A Multi-Parametric CFD Analysis of Multiphase Annular Flows for Oil and Gas Drilling Applications. Computers \& Chemical Engineering, 106, 645-661. https://doi.org/10.1016/j.compchemeng.2017.08.011

[2] Epelle, E.I. and Gerogiorgis, D.I. (2018) Transient and Steady State Analysis of Drill Cuttings Transport Phenomena under Turbulent Conditions. Chemical Engineering Research and Design, 131, 520-544. https://doi.org/10.1016/j.cherd.2017.11.023

[3] Epelle, E. and Gerogiorgis, D. (2019) Mixed-Integer Nonlinear Programming (MINLP) for Production Optimization of Naturally Flowing and Artificial Lift Wells with Routing Constraints. Chemical Engineering Research and Design, 152, 134-148. https://doi.org/10.1016/j.cherd.2019.09.042

[4] Abdallah, A.G. (2011) A New Productivity Index Formula for ESP Lifted Wells. Proceedings of the SPE Middle East Oil and Gas Show and Conference, Manama, 25-28 September 2011, SPE-141984-MS.

[5] Andersson, J.A.E., Gillis, J., Horn, G., Rawlings, J.B. and Diehl, M. (2019) CasADi-A Software Framework for Nonlinear Optimization and Optimal Control. Mathematical Programming Computation, 11, 1-36. https://doi.org/10.1007/s12532-018-0139-4

[6] Castro, V., Leite, D., Lemos, D., Marins, J., Pessoa, R. and Magalhaes, J. (2015) ESP Application on Heavy Oil in Peregrino Field. Proceedings of the SPE Artificial Lift Conference-Latin America and Caribbean, Salvador, 27-28 May 2015, SPE-173948MS. https://doi.org/10.2118/173948-MS

[7] Foss, B., Knudsen, B.R. and Grimstad, B. (2018) Petroleum Production Optimization-Astatic or Dynamic Problem? Computers \& Chemical Engineering, 114, 245-253. https://doi.org/10.1016/j.compchemeng.2017.10.009

[8] Takacs, G. (2011) How to Improve Poor System Efficiencies of ESP Installations Controlled by Surface Chokes. Journal of Petroleum Exploration and Production Technology, 1, 89-97. https://doi.org/10.1007/s13202-011-0011-9

[9] Güler, N.O. (2004) Production System Optimization for Submersible Pump Lifted Wells: A Case Study. Master's Thesis, Middle East Technical University, Ankara. 
[10] Mahgoub, I.S., Shahat, M.M. and Fattah, S.A. (2005) Overview of ESP Application in Western Desert of Egypt-Strategy for Extending Lifetime. The International Petroleum Technology Conference, Doha, 21-23 November 2005, IPTC-10142-MS. https://doi.org/10.2523/IPTC-10142-MS

[11] Sharma, R. and Glemmestad, B. (2013) Optimal Control Strategies with Nonlinear Optimization for an Electric Submersible Pump Lifted Oil Field. Modeling, Identification and Control, 34, 55-67. https://doi.org/10.4173/mic.2013.2.2

[12] Sharma, R. and Glemmestad, B. (2013) Modeling and Simulation of an Electric Submersible Pump Lifted Oil Field. International Journal of Petroleum Science and Technology, 8, 39-68.

[13] Krishnamoorthy, D., Bergheim, E.M., Pavlov, A., Fredriksen, M. and Fjalestad, K. (2016) Modelling and Robustness Analysis of Model Predictive Control for Electrical Submersible Pump Lifted Heavy Oil Wells. IFAC-PapersOnLine (DYCOPS), 49, 544-549. https://doi.org/10.1016/j.ifacol.2016.07.399

[14] Krishnamoorthy, D., Fjalestad, K. and Skogestad, S. (2019) Optimal Operation of Oil and Gas Production Using Simple Feedback Control Structures. Control Engineering Practice, 91, Article ID: 104107.

https://doi.org/10.1016/j.conengprac.2019.104107

[15] Gupta, V. and Grossmann, I.E. (2012) Modelling and Computational Strategies for Optimal Development Planning of Offshore Oilfields under Complex Fiscal Rules. Industrial \& Engineering Chemistry Research, 51, 14438-14460. https://doi.org/10.1021/ie301482d

[16] Khor, C.S., Elkamel, A. and Shah, N. (2017) Optimization Methods for Petroleum Fields Development and Production Systems: A Review. Optimization and Engineering, 18, 907-941. https://doi.org/10.1007/s11081-017-9365-2

[17] Tavallali, M.S., Karimi, I.A. and Baxendale, D. (2016) Process Systems Engineering Perspective on the Planning and Development of Oil Fields. AIChE Journal, 62, 25862604. https://doi.org/10.1002/aic.15209

[18] Kritsadativud, P., Jafarpour, B. and Ekkawong, P. (2015) Fast Production Optimization with Decline Curve Analysis under Facility Constraints: A Field Case Study. SPE Western Regional Meeting, Garden Grove, 27-30 April 2015, SPE-174039-MS. https://doi.org/10.2118/174039-MS

[19] Hoffmann, A. and Stanko, M.E. (2016) Real-Time Production Optimization of a Production Network with ESP Boosted Wells: A Case Study. The SPE Middle East Artificial Lift Conference and Exhibition, Manama, 30 November-1 December 2016, SPE-184189-MS. https://doi.org/10.2118/184189-MS

[20] Gunnerud, V., Conn, A. and Foss, B. (2013) Embedding Structural Information in Simulation-Based Optimization. Computers \& Chemical Engineering, 53, 35-43. https://doi.org/10.1016/j.compchemeng.2013.02.004

[21] Bates, R., Cosad, C., Fielder, L., Kosmala, A., Hudson, S., Romero, G. and Shamnugam, V. (2004) Taking the Pulse of Producing Wells ESP Surveillance. Oilfield Review, 16, 16-25. https://doi.org/10.7748/ns.19.16.25.s34

[22] Stanko, M. and Golan, M. (2015) Exploring the Potential of Model-Based Optimization in Oil Production Gathering Networks with ESP-Produced High Water Cut Wells. 17 th International Conference on Multiphase Technology, Cannes, 10 June 2015, BHR-2015-J2.

[23] Baker Hughes Centrilift (2008) Submersible Pump Handbook, Eight Edition Version 2 .

[24] Hollund, B.S. (2010) Artificial Lift-Electrical Submerged Pump, Best Practice and Future Demands within Subsea Applications. Master's Thesis, University of Stavan- 
ger, Stavanger.

[25] Dupoiron, M. (2017) The Effect of Gas on Multi-Stage Mixed-Flow Centrifugal Pumps. Ph.D. Thesis, University of Cambridge, Cambridge.

\section{Nomenclature}

ESP: Electrical submersible pump;

ESPs: Electrical submersible pumps;

CBM: Coalbed methane;

GAP: General Allocation Package. 